\title{
EGFR mutation status yield from bronchoalveolar lavage in patients with primary pulmonary adenocarcinoma compared to a venous blood sample and tissue biopsy
}

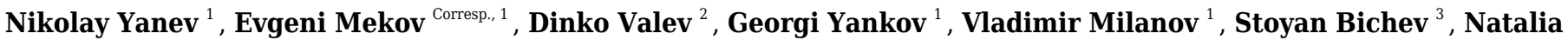 \\ Gabrovska $^{1}$, Dimitar Kostadinov ${ }^{1}$ \\ 1 Department of Pulmonary Diseases, Medical University - Sofia, Sofia, Bulgaria \\ 2 University Hospital "St. Ioan Krustitel”, Sofia, Bulgaria \\ 3 National Genetics Laboratory, Medical University - Sofia, Sofia, Bulgaria \\ Corresponding Author: Evgeni Mekov \\ Email address: dr_mekov@abv.bg
}

Background. In recent years, there has been a revolution in the genomic profiling and molecular typing of lung cancer. A key oncogene is the epidermal growth factor receptor (EGFR). The gold standard for determining EGFR mutation status is tissue biopsy, where a histological specimen is taken by a bronchoscopic or surgical method (transbronchial biopsy, forceps biopsy, etc.). However, in clinical practice the tissue sample is often insufficient for morphological and molecular analysis. Bronchoalveolar lavage is a validated diagnostic method for pathogenic infections in the lower respiratory tract, yet its diagnostic value for oncogenic mutation testing in lung cancer has not been extensively investigated. This study aims to compare the prevalence of EGFR mutation status in bronchoalveolar lavage and peripheral blood referring to the gold standard - tissue biopsy in patients with primary lung adenocarcinoma.

Methods. Twenty-six patients with adenocarcinoma were examined for EGFR mutation from tissue biopsy, peripheral blood sample and bronchoalveolar lavage.

Results. 13 patients had wild type EGFR and the other 13 had EGFR mutation. EGFR mutation from a peripheral blood sample was identified in $38.5 \%$ (5/13) of patients, whereas EGFR mutation obtained from bronchoalveolar lavage (BAL) was identified in $92.3 \%(12 / 13)$. This study demonstrates that a liquid biopsy sample for EGFR status from BAL has a higher sensitivity compared to a venous blood sample. 
1 EGFR mutation status yield from bronchoalveolar lavage in patients with

2 primary pulmonary adenocarcinoma compared to a venous blood sample and

$4 \quad$ Nikolay Yanev ${ }^{1}$, Evgeni Mekov ${ }^{1}$, Dinko Valev², Georgi Yankov', Vladimir

$5 \quad$ Milanov $^{1}$, Stoyan Bichev ${ }^{3}$, Natalia Gabrovska ${ }^{1}$, Dimitar Kostadinov ${ }^{1}$

6 1. Department of Pulmonary Diseases, Medical University - Sofia, Medical Faculty, Sofia, 7 Bulgaria

8 2. University Hospital "St. Ioan Krustitel”, Sofia, Bulgaria

9 3. University Hospital of Obstetrics and Gynecology "Maichin Dom", Medical University - Sofia, 10 National Genetics Laboratory, Sofia, Bulgaria

*Corresponding author:

13 Evgeni Mekov

MHATPD 'Sveta Sofia', Sofia, Bulgaria. Tel. +359 888320 476, e-mail:

\section{5 evgeni.mekov@gmail.com}

\section{Abstract}

Background. In recent years, there has been a revolution in the genomic profiling and molecular typing of lung cancer. A key oncogene is the epidermal growth factor receptor (EGFR). The gold standard for determining EGFR mutation status is a tissue biopsy, where a histological specimen is taken by a bronchoscopic or surgical method (transbronchial biopsy, forceps biopsy, etc.). However, in clinical practice 
23 the tissue sample is often insufficient for morphological and molecular analysis.

24 Bronchoalveolar lavage is a validated diagnostic method for pathogenic infections

25 in the lower respiratory tract, yet its diagnostic value for oncogenic mutation testing

26 in lung cancer has not been extensively investigated. This study aims to compare the

27 prevalence of EGFR mutation status in bronchoalveolar lavage and peripheral blood

28 referring to the gold standard - tissue biopsy in patients with primary lung

29 adenocarcinoma.

30 Methods. Twenty-six patients with adenocarcinoma were examined for EGFR

31 mutations from tissue biopsy, peripheral blood sample and bronchoalveolar lavage.

32 Results. 13 patients had wild type EGFR and the other 13 had an EGFR mutation.

33 EGFR mutations from a peripheral blood sample were identified in $38.5 \%(5 / 13)$ of

34 patients, whereas an EGFR mutation obtained from bronchoalveolar lavage (BAL)

35 was identified in $92.3 \%(12 / 13)$. This study demonstrates that a liquid biopsy sample

36 for EGFR status from BAL has a higher sensitivity compared to a venous blood

37 sample.

Keywords: adenocarcinoma, lung cancer, EGFR, liquid biopsy, bronchoalveolar

40 lavage

\section{Introduction}


In recent years, there has been a revolution in the genomic profiling and

44 molecular typing of lung cancer. Full genomic profiling is recommended for the

45 selection of the optimal personalized therapy.

The epidermal growth factor receptor (EGFR) is a key oncogene for targeted

47 therapy along with anaplastic lymphoma kinase (ALK), v-Raf murine sarcoma viral

48

49

50

51

52

53

54

55

56

57

58

59

60

61

62 oncogene homolog B (BRAF), MET, and ROS. Treatment with first and secondgeneration EGFR tyrosine kinase inhibitors such as erlotinib, gefitinib, and afatinib is the standard of care in patients with sensitive mutations (Exon 19 Deletions, L858R) in the kinase domain of the EGFR gene [1].

The gold standard for determining the EGFR mutation status is tissue biopsy, where a histological specimen is taken by a bronchoscopic or surgical method (transbronchial biopsy, forceps biopsy, etc.). However, in clinical practice the tissue sample is often insufficient for morphological and molecular analysis [24]. sample available is either cytological material or a limited amount of tissue [5]. What is more, a biopsy is an invasive procedure associated with a higher risk for complications [6] and the results of genetic biopsy examinations of a biopsy sample from a single tumor site is not a reflection of tumor heterogeneity. Hence, the clinician is unable to observe the molecular resistance in the treatment process [7]. 
63 On the other hand, the isolation of free circulating peripheral blood tumor DNA

64 (ctDNA) known as 'liquid biopsy' is a non-invasive, alternative method to tissue

65 biopsy for performing molecular screening in patients suitable for targeted therapy.

66 The sensitivity and specificity of the method vary depending on the research

67 platform and the quality of the test material [8].

69 Aim

This study aims to compare the prevalence of EGFR mutation status in bronchoalveolar lavage samples with the prevalence in peripheral blood, referring to the gold standard - tissue biopsy, in patients with primary lung adenocarcinoma.

\section{Material and Methods}

We assessed all patients scheduled for bronchoscopy in our hospital between

October 2018 and August 2019. The patients were further evaluated according to the protocol if lung adenocarcinoma was confirmed. The exclusion criteria were in concordance with the standard contraindications for a bronchoscopy. The research protocol was approved by the Ethical Committee of the Medical University Sofia (approval letter 1601/15.04.2019). Written informed consent was obtained from all patients included in this study before they underwent bronchoscopy. 
82 Flexible bronchoscopy was performed in all patients under local anesthesia

83 with lidocaine ( $10 \%$ lidocaine spray) in nasal passages, followed by nasal instillation

84 of a $2 \%$ solution during the procedure through the working channel, achieving a total

85 dose of up to $8 \mathrm{mg} / \mathrm{kg}$. The bronchoscopies were performed with Olympus Exera II

CV-180, Pentax EPK-1000 and Fujinon EB-530T optical and video devices. The

87 biopsy techniques were forceps biopsy, transbronchial biopsy with or without

88 fluorographic control, based on preliminary CT data, and bronchoalveolar lavage

89 (BAL).

BAL was performed under local anesthesia after an examination of the tracheobronchial tree. The $0.9 \%$ saline, which was used for the procedure, was preheated to body temperature. The fluid was instilled with syringes through the biopsy canal by repeatedly injecting 20-60 $\mathrm{ml}$ (usually 4 or 5 times) to a total volume of 100-240 ml. After each administration the fluid was aspirated back and the aspirated volume amounted to $40-70 \%$ of the initially administered one. solution, whereas the cytological materials were applied to a glass slide - a total of 4 pieces. BAL and plasma samples were frozen immediately at $-10^{\circ} \mathrm{C}$. No BAL sample underwent centrifugation. 
The histological materials were evaluated by a clinical pathologist through via

102 light microscopy and stained with hematoxylin-eosin. Additional 103 immunohistochemistry and genetic tests were performed if necessary. In cases where adenocarcinoma was confirmed from a tissue sample, the frozen plasma and BAL samples were sent for DNA analysis in the National Genetic Laboratory. There, DNA extraction was performed with QiaAmp Circulating Nucleic Acid kit for plasma and BAL samples. For Formalin-Fixed ParaffinEmbedded Tissue (FFPET) samples, we used the QIAamp DNA FFPE Tissue Kit.

EGFR genotyping was performed via PCR with therascreen EGFR Plasma or tissue RGQ PCR Kits provided by QIAGEN ${ }^{\circledR}$. The platform used was the Rotor-Gene Q PCR. Prior to the beginning of the project, a validation of the assay for lavage samples was conducted. DNA was extracted from six BAL samples of patients with an already determined EGFR status. DNA extraction was performed with the Qiavac24 system. Four of the patients had wtEGFR and from the remaining two, one

115 was positive for an Exon 19 deletion and one for L858R point mutation. The 116 obtained DNA concentration was in the range of $7.5-25 \mathrm{ng} / \mu 1$. PCR analysis of the samples was performed according to the IFU of the therascreen ${ }^{\circledR}$ EGFR Plasma RGQ PCR Kit Handbook (January 2019). All samples were amplified in three receptions. EGFR mutations were confirmed in both samples and in all replicates.

120 Detected $\mathrm{Ct}$ and delta $\mathrm{Ct}$ values were in the range described in the manual. 
122 Results

123 Between October 2018 and August 2019, 140 bronchoscopies were 124 performed. In 112 patients $(80 \%)$ the histopathological analysis of the acquired 125 tissue samples confirmed the presence of a lung malignancy (Table 1). Due to the 126 limited size of the patient sample, the prevalence of adenocarcinoma and EGFR 127 mutations deviates to a certain extent from established data. Adenocarcinoma was 128 present in $23.2 \%(26 / 112)$ of the patients with lung cancer which was verified 129 through immunohistochemistry on tissue samples. 13 patients were female and 13 130 were male with a mean age of 63.3 years. The localization of the tumor was evenly 131 spread with 13 left-sided, 12 right-sided, and 1 bilateral (without distinctive primary 132 lesion) cases. The average maximum diameter of the tumors was $5.53 \mathrm{~cm}(\min 2.3$ $133 \mathrm{~cm}$, max $12.4 \mathrm{~cm}$ ). Interestingly, a visible, intralumenal tumor was present in 23/26 134 patients $(88.5 \%)$.

Table 1. Patient demographics

The groups did not differ with respect to the age and tumor maximum diameter (Table 2). However, there were significantly more women with EGFR mutation $(77 \%, 10 / 13)$ as opposed to men $(23 \%, 3 / 13, \mathrm{p}=0.017)$. 
142 Table 2. Patient demographics and tumor characteristics

143

144

Thirteen patients had wild type EGFR and the other 13 had EGFR mutation.

145

EGFR mutation from a peripheral blood sample was identified in $38.5 \%(5 / 13)$

146

patients, whereas EGFR mutation obtained from BAL was identified in $92.3 \%$

147

(12/13) (Table 3). In one case in both liquid biopsy samples (BAL and plasma) an

additional T790M mutation was detected, along with a deletion in exon 19, which

was not established in the corresponding FFPET sample (patient 8). Neither BAL,

not blood sample confirmed EGFR mutation in 1 patient which is positive only from

151

a tissue sample (Table 4).

152

153

Table 3. Confirmation of EGFR mutation in BAL and blood samples

154

155 Table 4. EGFR mutation status in the individuals

156

157

\section{Discussion}

158

The present study compares the detectability of activating EGFR mutations in

159

BAL and blood plasma samples, compared to the result from the tissue biopsy. In

this study, BAL was found to be more sensitive with regards to detecting an EGFR 
161 mutation compared to liquid biopsy $-92.3 \%$ vs. $38.5 \%$ in the same patients. This

162 result is consistent with the published data in other studies [9]. Moreover, in two

163 cases, BAL provides additional data about the mutational status, compared to

164 histology specimens (table 4, case numbers 8 , and 12 ), a fact most likely attributable

165 to tumor heterogeneity and tissue sampling process. Thus, more information and

166 more treatment choices may be available for the patients.

167 The rapid growth of tumor cells leads to apoptosis and necrosis. In some cases,

168 the amount of post-apoptotic residues and fragments exceeds phagocytic clearance.

169 This leads to the presence of a certain concentration of tumor cell debris, including

170 ctDNA, into the systemic circulation, where they are isolated from [10]. The use of

171 blood plasma for the detection of ctDNA is a non-invasive method, which represents

172 a feasible option in cases where tissue biopsy is not possible. However, it requires

173 the use of more sensitive diagnostic methods due to the very small amount of ctDNA

174 in plasma $[11,12]$. The results from a meta-analysis of 3110 patients showed that the

175 blood plasma liquid biopsy has $62 \%$ sensitivity and specificity of $95 \%$. Although the

176 sensitivity is lower in comparison with tissue biopsy, the authors state that ctDNA

177 testing is an effective diagnostic method for EGFR mutation detection with a very

178 high specificity [13].

179 A limited number of studies have been directed to the study of EGFR

180 mutational status from bronchoalveolar lavage. Bronchoalveolar lavage has some 
181 advantages - a semi-invasive method associated with a lower risk complications due

182 to the procedure, compared to tissue biopsy [14], even at more advanced stages of

183 the disease. Furthermore, since more cellular debris, resulting from tumor apoptosis

184 and necrosis, accumulate in a localized area in the lungs before entering the vascular

185 system, the ctDNA concentration would be higher in bronchoalveolar lavage (BAL)

186 than in plasma $[15,16]$. Hence, one could make an assumption that the probability to

187 identify the EGFR mutation status would be higher.

188 Also, in bronchoalveolar lavage, it is possible to have ctDNA fragments

189 from different regions of the tumor surface (tumor heterogeneity), which increases

190 the chance of identifying mutations from multiple tumor locations.

Schmidt et al. isolated free tumor lavage DNA in $46.7 \%(14 / 30)$ of patients

192

[17]. In Park et al., 91.7\% of BAL established EGFR status compared to tumor

biopsy using a high-sensitivity method [9].

194

Currently, re-biopsy is the gold standard for the detection of EGFR mutations in patients with unknown status after negative tissue sampling. Unfortunately, rebiopsy is not always feasible due to many reasons (comorbidity and condition of the patient, difficulties in obtaining biopsy specimens). In this context, liquid biopsy could be an effective solution. Results from individual studies are variable, and even

199 though its sensitivity is approximately $62 \%$ [13], ctDNA is a very specific and 200 effective biomarker. Furthermore, establishing EGFR status from BAL is a viable 
201 and safe procedure as well, especially in impaired patients with promising results.

202 Since the BAL samples didn't undergo centrifugation, one could not say for sure if

203 the positive result derives from ctDNA or from tumor cells, present in the sample.

204 Lastly, one should not underestimate the fact that $88.5 \%$ of the patients with 205 adenocarcinoma $(23 / 26)$ had visible intralumenal tumors. The correlation of the

206 BAL sensitivity with the presence of intralumenal lesions needs further 207 investigating, as BAL could turn out to be a workable diagnostic tool in such clinical 208 cases.

209

210

\section{Conclusion}

211

We managed to demonstrate a higher sensitivity of BAL to venous blood

212 liquid biopsy in establishing an EGFR mutation in the patient population, which

213 participated in this study. This could facilitate a diagnosis with a minimally invasive

214 method and lower risk for the patient with comparable results to FFPET in this

215 limited group of patients.

216

217

References:

218

219

220

1. Novello S, Barlesi F, Califano R, Cufer T, Ekman S, Levra MG, Kerr K, Popat S, Reck M, Senan S, Simo GV, Vansteenkiste J, Peters S; ESMO Guidelines Committee. Metastatic non-small-cell lung cancer: ESMO Clinical Practice 
221 Guidelines or diagnosis, treatment and follow-up. Ann Oncol 2016;27:v1$222 \quad \mathrm{v} 27$.

223

224

225

226

227

228

229

230

231

232

233

234

235

236

237

238

239

240

241

242

243

244

245

246

2. Coghlin C, Smith L, Bakar S, Stewart KN, Devereux GS, Nicolson MC, Kerr KM. Quantitative Analysis of Tumor in Bronchial Biopsy Specimens. J Thorac Oncol 2010;5(4):448-52.

3. Rivera MP, Mehta AC, Wahidi MM. Establishing the Diagnosis of Lung Cancer: Diagnosis and Management of Lung Cancer, 3rd ed: American College of Chest Physicians Evidence-Based Clinical Practice Guidelines. Chest 2013;143(5 Suppl):e142S-e165S.

4. Weber B, Meldgaard P, Hager H, Wu L, Wei W, Tsai J, Khalil A, Nexo E, Sorensen BS. Detection of EGFR mutations in plasma and biopsies from nonsmall-cell lung cancer patients by allele-specific PCR assays. BMC Cancer 2014;14:294.

5. Bubendorf L, Lantuejoul S, de Langen AJ, Thunnissen E. Nonsmall cell lung carcinoma: diagnostic difficulties in small biopsies and cytological specimens: Number 2 in the Series "Pathology for the clinician" Edited by Peter Dorfmüller and Alberto Cavazza. Eur Respir Rev 2017;26(144):170007.

6. Lorenz J, Blum M. Complications of percutaneous chest biopsy. Semin Intervent Radiol 2006;23(2):188-93.

7. Gerlinger M, Rowan AJ, Horswell S, Math M, Larkin J, Endesfelder D, Gronroos E, Martinez P, Matthews N, Stewart A, Tarpey P, Varela I, Phillimore B, Begum S, McDonald NQ, Butler A, Jones D, Raine K, Latimer C, Santos CR, Nohadani M, Eklund AC, Spencer-Dene B, Clark G, Pickering L, Stamp G, Gore M, Szallasi Z, Downward J, Futreal PA, Swanton C. Intratumor heterogeneity and branched evolution revealed by multiregion sequencing. N Engl J Med 2012;366:883-92. 
247

248

249

250

251

252

253

254

255

256

257

258

259

260

261

262

263

264

265

266

267

268

269

270

271

272

8. Sacher AG, Komatsubara KM, Oxnard GR. Application of Plasma Genotyping Technologies in Non-Small Cell Lung Cancer: A Practical Review. J Thorac Oncol 2017;12:1344-56.

9. Park S, Hur J, Lee K, Lee JC, Rho JK, Shin SH, Choi CM. Assessment of EGFR mutation status using cell-free DNA from bronchoalveolar lavage fluid. CCLM 2017;55(10):1489-95.

10. Diehl F, Schmidt K, Choti MA, Romans K, Goodman S, Li M, Thornton K, Agrawal N, Sokoll L, Szabo SA, Kinzler KW, Vogelstein B, Diaz LA Jr. Circulating mutant DNA to assess tumor dynamics. Nat Med 2008;14:98590.

11. Zachariah RR, Schmid S, Buerki N, Radpour R, Holzgreve W, Zhong X. Levels of circulating cell-free nuclear and mitochondrial DNA in benign and malignant ovarian tumors. Obstet Gynecol 2008;112:843-50.

12. Zanetti-Dallenbach RA, Schmid S, Wight E, Holzgreve W, Ladewing A, Hahn S, Zhong XY. Levels of circulating cell-free serum DNA in benign and malignant breast lesions. Int J Biol Markers 2007;22:95-9.

13. Qiu M, Wang J, Xu Y, Ding X, Li M, Jiang F, Xu L, Yin R. Circulating Tumor DNA Is Effective for the Detection of EGFR Mutation in Non-Small Cell Lung Cancer: A Meta-analysis. Cancer Epidemiol Biomarkers Prev 2014;24(1):206-12.

14. Herth F, Shah P, Gompelmann D. European Respiratory Society Monograph: Interventional Pulmonology. Volume 78, 2017.

15. Jahr S, Hentze H, Englisch S, Hardt D, Fackelmayer FO, Hesch RD, Knippers R. DNA fragments in the blood plasma of cancer patients: quantitations and evidence for their origin from apoptotic and necrotic cells. Cancer Res 2001;61:1659-65. 
273

274

275

276

277

278

279
16. Stroun M, Lyautey J, Lederrey C, Olson-Sand A, Anker P. About the possible origin and mechanism of circulating DNA apoptosis and active DNA release. Clin Chim Acta 2001;313:139-42.

17. Schmidt B, Carstensen T, Engel E, Jandrig B, Witt C, Fleischhacker M. Detection of cell-free nucleic acids in bronchial lavage fluid supernatants from patients with lung cancer. Eur J Cancer 2004;40:452-60. 
280 Table 1. Patient demographics

\begin{tabular}{|l|l|}
\hline Characteristic & Value \\
\hline Age, years, mean $( \pm \mathrm{SD})$ & $64,4( \pm 8.4)$ \\
\hline Male gender, n $(\%)$ & $100(71,4)$ \\
\hline Histology & $\mathbf{n}(\%)$ \\
\hline - Adenocarcinoma & $26(18,6)$ \\
\hline - Squamous cell carcinoma & $36(25,7)$ \\
\hline - Non-squamous cell carcinoma & $23(16,4)$ \\
\hline - Small cell carcinoma & $27(19,3)$ \\
\hline - Chronic nonspecific inflammation & $18(12,9)$ \\
\hline - Unidentified lung disease & $10(7,1)$ \\
\hline
\end{tabular}

281 
282 Table 2. Patient demographics and tumor characteristics

\begin{tabular}{|l|c|c|}
\hline \multicolumn{1}{|c|}{ Characteristic } & $\begin{array}{c}\text { EGFR mutation group } \\
(\mathbf{n = 1 3 )}\end{array}$ & $\begin{array}{c}\text { Wild type EGFR group } \\
(\mathbf{n}=\mathbf{1 3})\end{array}$ \\
\hline Mean age, years & 62 & 64,5 \\
\hline Male gender, $\mathrm{n}$ & 3 & 10 \\
\hline Tumor localization & & 6 \\
\hline$-\quad$ Left $(\mathrm{n})$ & 7 & 7 \\
\hline- Right $(\mathrm{n})$ & 5 & 0 \\
\hline- Both sides $(\mathrm{n})$ & 4,9 & 5,9 \\
\hline $\begin{array}{l}\text { Tumor diameter, mean } \\
(\mathrm{cm})\end{array}$ & 10 & 13 \\
\hline Visible tumor $(\mathrm{n})$ & & \\
\hline
\end{tabular}

283 
284 Table 3. Confirmation of EGFR mutation in BAL and blood samples

\begin{tabular}{|l|l|}
\hline & Confirmed EGFR mutation \\
\hline BAL sample & $12(92.3 \%)$ \\
\hline Blood plasma & $5(38.5 \%)$ \\
\hline Total patients & $13(100 \%)$ \\
\hline
\end{tabular}

285 
286 Table 4. EGFR mutation status in the individuals

\begin{tabular}{|r|l|l|l|}
\hline $\mathbf{N}$ & \multicolumn{1}{|c|}{$\boldsymbol{F F P E T}$} & \multicolumn{1}{|c|}{ BAL } & \multicolumn{1}{c|}{ Blood plasma } \\
\hline $\mathbf{1}$ & Exon 19 deletion & Exon 19 deletion & Wild type \\
\hline $\mathbf{2}$ & Exon 21 L858R & Exon 21 L858R & Wild type \\
\hline $\mathbf{3}$ & Exon 21 L858R & Wild type & Wild type \\
\hline $\mathbf{4}$ & Exon 19 deletion & Exon 19 deletion & Wild type \\
\hline $\mathbf{5}$ & Exon 21 L858R & Exon 21 L858R & Wild type \\
\hline $\mathbf{6}$ & Exon18 G719 & Exon 18 G719 & Exon 18 G719 \\
\hline $\mathbf{7}$ & Exon 19 deletion & Exon 19 deletion & Exon 19 deletion \\
\hline $\mathbf{8}$ & Exon 19 deletion & Exon 19 deletion/T790M* & Exon 19 deletion \\
\hline $\mathbf{9}$ & Exon 19 deletion & Exon 19 deletion & Exon 19 deletion \\
\hline $\mathbf{1 0}$ & Exon 19 deletion & Exon 19 deletion & Wild type \\
\hline $\mathbf{1 1}$ & Exon 19 deletion & Exon 19 deletion & Exon 19 deletion \\
\hline $\mathbf{1 2}$ & Exon 21 L858R & Exon 21 L858R/Exon 19 deletion* & Wild type \\
\hline $\mathbf{1 3}$ & Exon 19 deletion & Exon 19 deletion & Wild type \\
\hline
\end{tabular}


Table $\mathbf{1}$ (on next page)

Patient demographics 


\begin{tabular}{|l|l|}
\hline Characteristic & Value \\
\hline Age, years, mean $( \pm \mathrm{SD})$ & $64,4( \pm 8.4)$ \\
\hline Male gender, n $(\%)$ & $100(71,4)$ \\
\hline Histology & $\mathbf{n}(\%)$ \\
\hline - Adenocarcinoma & $26(18,6)$ \\
\hline - Squamous cell carcinoma & $36(25,7)$ \\
\hline - Non-squamous cell carcinoma & $23(16,4)$ \\
\hline - Small cell carcinoma & $27(19,3)$ \\
\hline - Chronic nonspecific inflammation & $18(12,9)$ \\
\hline - Unidentified lung disease & $10(7,1)$ \\
\hline
\end{tabular}

1 
Table 2 (on next page)

Patient demographics and tumor characteristics 


\begin{tabular}{|l|c|c|}
\hline \multicolumn{1}{|c|}{ Characteristic } & $\begin{array}{c}\text { EGFR mutation group } \\
(\mathbf{n = 1 3 )}\end{array}$ & $\begin{array}{c}\text { Wild type EGFR group } \\
(\mathbf{n = 1 3 )}\end{array}$ \\
\hline Mean age, years & 62 & 64,5 \\
\hline Male gender, $\mathrm{n}$ & 3 & 10 \\
\hline Tumor localization & & 6 \\
\hline$-\quad$ Left $(\mathrm{n})$ & 7 & 7 \\
\hline- Right $(\mathrm{n})$ & 1 & 0 \\
\hline - Both sides $(\mathrm{n})$ & 4,9 & 5,9 \\
\hline $\begin{array}{l}\text { Tumor diameter, mean } \\
(\mathrm{cm})\end{array}$ & 10 & 13 \\
\hline Visible tumor $(\mathrm{n})$ & & \\
\hline
\end{tabular}

1 
Table 3 (on next page)

Confirmation of EGFR mutation in BAL and blood samples 


\begin{tabular}{|l|l|}
\hline & Confirmed EGFR mutation \\
\hline BAL sample & $12(92.3 \%)$ \\
\hline Blood plasma & $5(38.5 \%)$ \\
\hline Total patients & $13(100 \%)$ \\
\hline
\end{tabular}

1 
Table 4 (on next page)

EGFR mutation status in the individuals

* - additional mutation 


\begin{tabular}{|r|l|l|l|}
\hline $\mathbf{N}$ & \multicolumn{1}{|c|}{ FFPET } & \multicolumn{1}{|c|}{ BAL } & \multicolumn{1}{c|}{ Blood plasma } \\
\hline $\mathbf{1}$ & Exon 19 deletion & Exon 19 deletion & Wild type \\
\hline $\mathbf{2}$ & Exon 21 L858R & Exon 21 L858R & Wild type \\
\hline $\mathbf{3}$ & Exon 21 L858R & Wild type & Wild type \\
\hline $\mathbf{4}$ & Exon 19 deletion & Exon 19 deletion & Wild type \\
\hline $\mathbf{5}$ & Exon 21 L858R & Exon 21 L858R & Wild type \\
\hline $\mathbf{6}$ & Exon18 G719 & Exon 18 G719 & Exon 18 G719 \\
\hline $\mathbf{7}$ & Exon 19 deletion & Exon 19 deletion & Exon 19 deletion \\
\hline $\mathbf{8}$ & Exon 19 deletion & Exon 19 deletion/T790M* & Exon 19 deletion \\
\hline $\mathbf{9}$ & Exon 19 deletion & Exon 19 deletion & Exon 19 deletion \\
\hline $\mathbf{1 0}$ & Exon 19 deletion & Exon 19 deletion & Wild type \\
\hline $\mathbf{1 1}$ & Exon 19 deletion & Exon 19 deletion & Exon 19 deletion \\
\hline $\mathbf{1 2}$ & Exon 21 L858R & Exon 21 L858R/Exon 19 deletion* & Wild type \\
\hline $\mathbf{1 3}$ & Exon 19 deletion & Exon 19 deletion & Wild type \\
\hline
\end{tabular}

1 Legend: * - additional mutation 